\title{
Review \\ Deep Brain Stimulation in Animal Models of Fear, Anxiety, and Posttraumatic Stress Disorder
}

\author{
Roman Reznikov', Mary Binko', José N Nobrega ${ }^{1,2}$ and Clement Hamani, ${ }^{*, 1,3}$ \\ 'Behavioural Neurobiology Laboratory, Campbell Family Mental Health Research Institute, Centre for Addiction and Mental Health, Toronto, ON, \\ Canada; '2Departments of Psychiatry, Pharmacology and Toxicology, and Psychology, University of Toronto, Toronto, ON, Canada; ${ }^{3}$ Division of \\ Neurosurgery, Toronto Western Hospital, Toronto, ON, Canada
}

\begin{abstract}
Although medications and psychotherapy are often effective for the treatment of posttraumatic stress disorder (PTSD), 20-30\% of patients do not respond to these conventional therapies. In psychiatry, DBS has been either approved or is currently under investigation for different disorders. At present, whether DBS may be used to treat PTSD remains unknown. Preclinical research may provide the scientific rationale for helping conceive and further improve such trials. Some of the animal models commonly used to date are more suitable for investigating mechanisms of anxiety and fear than the long-lasting behavior that characterized PTSD. That said, mechanisms and neurocircuits involved in paradigms such as fear conditioning and extinction share several common features with those of PTSD. In this article, we review preclinical studies in which electrical stimulation has been delivered to animal models of PTSD-like behavior. In those studies, commonly targeted regions were the basolateral amygdala, ventral striatum, hippocampus, and prefrontal cortex. Overall, stimulation delivered at high frequencies to most of these targets improved fear extinction and anxiety-like behavior. Though further research is certainly needed, promising findings from DBS studies in animals are encouraging and suggest a positive future perspective for the field.

Neuropsychopharmacology (2016) 4I, 28I0-28I7; doi:I0.1038/npp.2016.34; published online 23 March 2016
\end{abstract}

\section{INTRODUCTION}

Posttraumatic stress disorder (PTSD) is a debilitating psychiatric illness that can manifest after exposure to highly stressful or life-threatening events. Although medications and psychotherapy are often effective, $20-30 \%$ of patients do not respond to conventional therapies. In psychiatry, DBS has been approved for the treatment of patients with medically refractory obsessive-compulsive disorder and is under investigation for conditions, such as drug addiction, anorexia, and depression. To date, aside from a single case report (Langevin et al, 2015), there are no published studies on the use of DBS in PTSD. Preclinical research may provide the scientific rationale for designing and further improving such trials (Hamani and Temel, 2012).

Numerous animal models have been proposed to mimic PTSD-like states. Though all have caveats, fear conditioning/ extinction stands out as the most commonly studied paradigm.

In this article, we review preclinical experiments in which electrical stimulation has been delivered to animal models of PTSD-like behavior, particularly fear

* Correspondence: Professor C Hamani, Neuroimaging Research Section, Centre for Addiction and Mental Health, 250 College Street, Toronto, ON M5T IR8, Canada, Tel: + I 4I6 535850I, ext 4295, Fax: + | 416 6035298, E-mail: Clement.Hamani@camh.ca

Received 24 November 2015; revised II January 2016; accepted 27 January 2016; accepted article preview online 2 March 2016 conditioning/extinction. Potential mechanisms for the effects of DBS are postulated in light of studies using optogenetics.

\section{FEAR CONDITIONING AND EXTINCTION}

Pavlovian fear conditioning is a paradigm whereby an animal learns to associate a neutral cue (the conditioned stimulus (CS)) with an aversive stimulus (the unconditioned stimulus (US)). After repeat CS-US pairings, presentation of the CS alone elicits a conditioned response (CR). As fear conditioning is an important survival mechanism that enables an animal to learn from experience, it is highly conserved across species. In humans, fear conditioning has been suggested to have a key role in the pathogenesis of PTSD. Patients with this disorder are hypersensitive to aversive stimuli and present strong fear learning (eg, CRs are robustly reinforced; Eysenck, 1979).

Extinction of fear conditioning refers to the gradual reduction of conditioned fear responses after repeated presentations of CS alone. Rather than memory deletion or erasure, the process of extinction involves the learning of new memories that suppress the initially conditioned fear. Impaired fear extinction is a commonly reported aspect of PTSD (VanElzakker et al, 2014). During exposure therapy, patients are presented with trauma-related cues or stimuli that gradually increase in intensity within the context of a safe environment. Though multiple sessions reduce symptoms in some individuals, these are not as effective in severe 
PTSD, suggesting that refractory patients may have deficits in extinction learning (Foa, 2000).

\section{Neurocircuitry}

After decades of study, the neurocircuitry of classical conditioning/extinction has been well characterized. The main structures implicated are the amygdala, hippocampus, and prefrontal cortex (PFC). Anatomical subdivisions of these three main structures in rodents are presented in Figure 1.

The lateral nucleus of the amygdala (LA) is a central relay of sensory information from different cortical and subcortical regions. As such, it has a major role in the acquisition and expression of conditioned fear (Maren and Quirk, 2004), facilitating the formation of memories linking US and CS. From the LA, signals are propagated to the central nucleus (CE), which is largely composed of GABAergic neurons and can be further subdivided into lateral (CEl) and medial subdivisions (CEm). The CEm is a major output structure of the amygdala, projecting to brainstem and hypothalamic areas involved in different aspects of the fear reaction, such as freezing behavior, autonomic, and endocrine responses. The hippocampus provides a contextual framework for the conditioned experience (VanElzakker et al, 2014), whereas the prelimbic cortex (PL) is important for the processing of transient incoming information and the generation of more stable patterns of fear response (Burgos-Robles et al, 2009).

The process of forming extinction memories is mediated by structures and nuclei (eg, within the PFC and amygdala) that are somewhat different from those involved in fear conditioning (Figure 2). Within the PFC, a dissociable role has been described between the infralimbic cortex (IL) and PL (Sierra-Mercado et al, 2011). During extinction, the former inhibits (Laurent and Westbrook, 2009; Vidal-Gonzalez et al, 2006), while the latter facilitates the behavioral expression of conditioned fear (Burgos-Robles et al, 2009; Vidal-Gonzalez et al, 2006). In fact, extinction learning seems to be dependent on plasticity within the IL, specifically that involving brain-derived neurotrophic factor (BDNF) (Andero and Ressler, 2012). Amygdala nuclei that are important in extinction include the intercalated cell cluster (ITC) and the basolateral nucleus (BLA). The ITC is comprised of GABAergic cells situated between the BLA and CE. These groups receive excitatory input from BLA and IL, sending inhibitory projections to CE. During extinction training, IL inputs activate the ITC, which subsequently inhibit the CE and reduce fear responses. Context-dependent fear renewal seems to depend on the integrity of the ventral hippocampus.

\section{DEEP BRAIN STIMULATION}

With a proven track record of alleviating symptoms in a variety of movement disorders, investigators have turned to testing the effectiveness of DBS in severe cases of treatmentrefractory psychiatric disorders. In the past decade, several studies have been conducted, ranging from small case reports to large multi-center placebo-controlled clinical trials, to study the safety and efficacy of DBS for treating obsessivecompulsive disorder, addiction, anorexia, and depression.

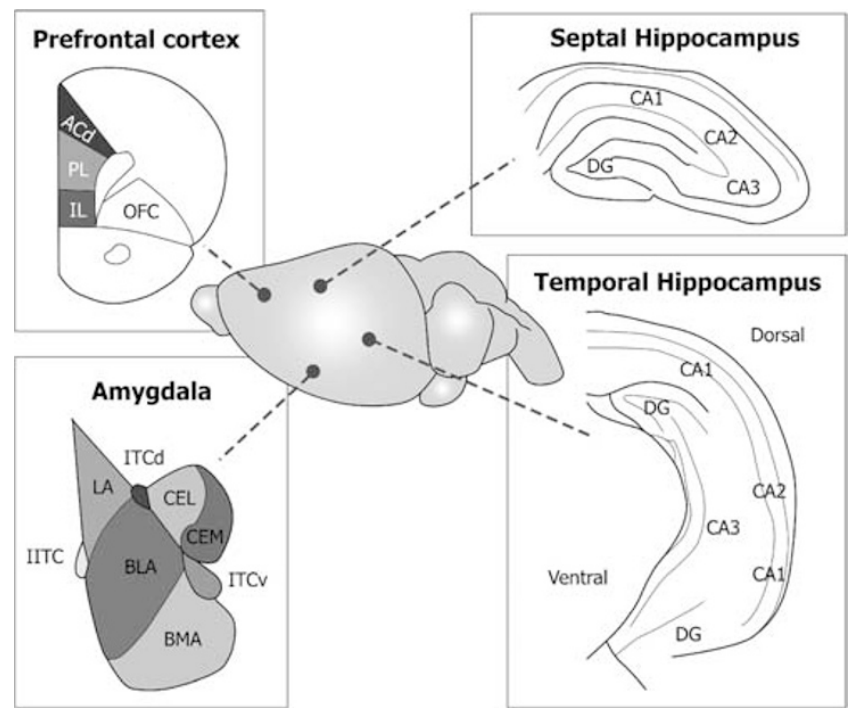

Figure I Schematic illustration of coronal sections of the rodent prefrontal cortex, amygdala, and hippocampus. Prefrontal regions in rodents may be divided into ventromedial prefrontal cortex and orbitofrontal cortex (OFC). The former is composed of the infralimbic cortex (IL), prelimbic cortex (PL), and anterior cingulate cortex (ACd). Amygdala nuclei shown in midsagittal views include the lateral nucleus ( $L A)$; basolateral nucleus (BLA); basomedial nucleus (BMA); lateral region of central nucleus (CEL); medial region of central nucleus (CEM); and lateral, dorsal, and ventral intercalated cell clusters (ITCS). The hippocampus is represented in more septal (anterior) and temporal (posterior) regions. The latter may be subdivided into dorsal and ventral. Hippocampal subfields include the dentate gyrus (DG), CAI, CA2, and CA3.

As of now, no clinical trials using deep brain stimulation in PTSD have been completed, though a case report has been recently published (Langevin et al, 2015).

In animal models, electrical stimulation has long been used to probe the behavioral and cognitive roles of various brain structures. The technique, however, lacks specificity: various neural elements (eg, cell bodies, dendrites, axons, and glia) are stimulated at the same time, current may spill into adjacent regions, and stimulation can influence structures at a distance from the target. As a result, the number of preclinical behavioral studies using electrical stimulation declined in the late 1970s and 1980s. This scenario began to change approximately 25 years ago, when DBS was reborn as a surgical technique for treating movement disorders. From that point on, investigators have once again used experimental animals to further understand mechanisms of DBS and explore new therapeutic applications/targets.

Overall, single pulses or trains of low-frequency extracellular cathodic stimulation lead to cell depolarization. Provided a certain membrane potential is reached, this culminates with the firing of action potentials. As a general rule, all neural elements are excited by stimulation but the time and current intensity required to excite axonal pathways, particularly large myelinated fibers, is 5-10-fold lower than those for dendrites or cell bodies (Ranck, 1975). When short-duration pulses are used, each stimulus is followed by an action potential.

Mechanisms for the effects of DBS at frequencies commonly used in clinical practice (ie, $130-185 \mathrm{~Hz}$ ) are somewhat distinct from the ones described above. At such 


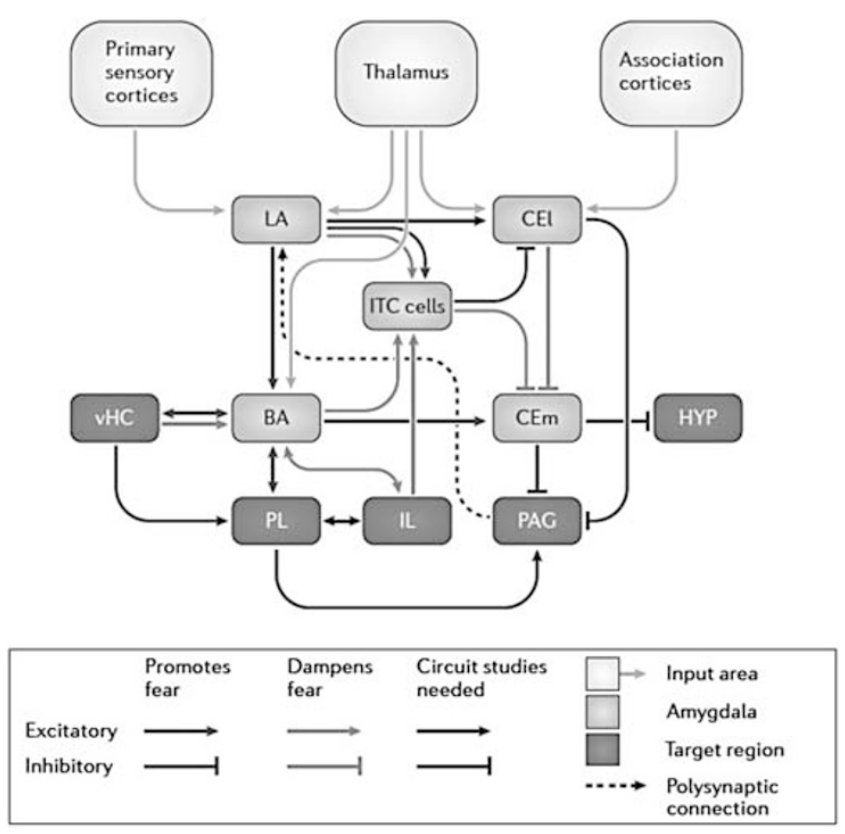

Figure 2 The fear and extinction network. Fear states are mediated by long-range excitatory and inhibitory connections between multiple brain areas. Several amygdala nuclei receive sensory input from cortical and thalamic centers and are major sites of fear-related neuronal plasticity. This plasticity is modulated by reciprocal connections between the basal amygdala (BA) and the ventral hippocampus ( $\mathrm{VHC}$ ), as well as between the $\mathrm{BA}$ and the prelimbic cortex (PL). In turn, central nuclei of the amygdala project to hypothalamic and brainstem centers to promote fear behavior. Extinction of fear is mediated by different circuit elements within the same structures. Input from the infralimbic cortex (IL) to the BA and to the intercalated (ITC) cells is instrumental in dampening fear output from lateral central amygdala (CEI) nuclei to the hypothalamus (HYP) and the periaqueductal gray (PAG). CEm, medial central amygdala; LA, lateral amygdala. Reprinted from Tovote et al (2015), by permission from Macmillan Publishers Ltd.

high frequencies, neuronal populations in the vicinity of the electrodes become functionally inactive (Lozano et al, 2002). This has been largely attributed to a process called depolarization block, characterized by a state in which cells undergo depolarization with an almost complete abolishment of spontaneous action potentials (Florence et al, 2015) (Figure 3a). In structures that receive prominent GABAergic projections, stimulation-induced GABA release from presynaptic terminals may also contribute to a functional target inactivation (Moser et al, 2003).

Another commonly proposed mechanism underlying the effects of high-frequency DBS is the excitation of fiber pathways in the vicinity of the electrodes (afferent and efferent projections from targeted regions as well as fibers en passant; Lozano et al, 2002). This is of importance, as the anterograde and retrograde propagation of action potentials along such structures may influence the physiology of brain regions projecting to or receiving projections from the original stimulation site (Figure 3b). Metabolic, electrophysiological, and neurochemical changes in structures at a distance from the target have all been suggested to contribute to the mechanisms of DBS in different applications of this therapy (Hamani and Temel, 2012; Hashimoto et al, 2003). It is worth mentioning, however, that the behavioral consequences of exciting axonal pathways may not always be positive, as physiological rhythms may be supplanted by a stimulation-induced tonic-firing pattern.

Not as explored in preclinical studies are the chronic consequences of DBS. These are fairly important as stimulation in humans is applied continuously for years. Plastic changes such as long-term potentiation and increases in neurotrophin levels and neurogenesis have all been described after electrical stimulation and may underlie some of the behavioral consequences of DBS (Hamani and Temel, 2012).

\section{PRECLINICAL STUDIES OF DBS ON FEAR AND ANXIETY}

In preclinical models, electrical stimulation has been delivered to various structures either to study its behavioral consequences or to understand the role of specific regions in mechanisms of fear conditioning/extinction and anxiety. Targets explored include the BLA, ventral striatum (VS), hippocampus, and PFC. A summary of some of these studies is provided below. Details of target and stimulation parameters may be found in Table 1.

\section{Basolateral Amygdala}

Investigators examining BLA DBS have mainly stimulated rats prior to defensive burying. This is an innate rodent behavior in which animals tend to bury objects considered to be dangerous, threatening, or associated with an unpleasant experience. Overall, treatments that reduce defensive burying are regarded as anxiolytic. In an initial study using a paradigm in which animals were to cover an energized shocking rod (Saldivar-Gonzalez et al, 2003), stimulation was delivered in a single session prior to behavioral testing at three different current intensities: 75,150 , or $300 \mu \mathrm{A}$. The authors found a decreased level of burying in animals receiving 150 and $300 \mu \mathrm{A}$. However, when stimulation was given at this latter setting, animals touched the rod and received shocks significantly more often, suggesting that the recorded effect was not exclusively due to a reduction in anxiety. Based on this finding and because animals receiving $150 \mu \mathrm{A}$ also had a decrease in the number of crossings in an elevated plus maze, this was considered to be an optimal stimulation parameter (Saldivar-Gonzalez et al, 2003). A notable result of that study was that high currents appeared to be toxic, being associated with the development of epileptiform afterdischarges (Saldivar-Gonzalez et al, 2003). In a second series of studies, rats exposed to a conspicuous object (a ball) were given a session of inescapable shocks (Langevin et al, 2010; Stidd et al, 2013). Thereafter, BLA DBS or sham treatment was administered for 1 week. DBS-treated rats spent significantly less time burying the ball than sham controls (Langevin et al, 2010), an effect that had a larger magnitude than that recorded with the antidepressant paroxetine (Stidd et al, 2013). In another study (Sui et al, 2014), chronic high-frequency BLA DBS administered following conditioning reduced the retrieval of tone but not contextual fear memory. This was attributed to a DBS-induced decrease in BLA plasticity during fear conditioning (Sui et al, 2014). 


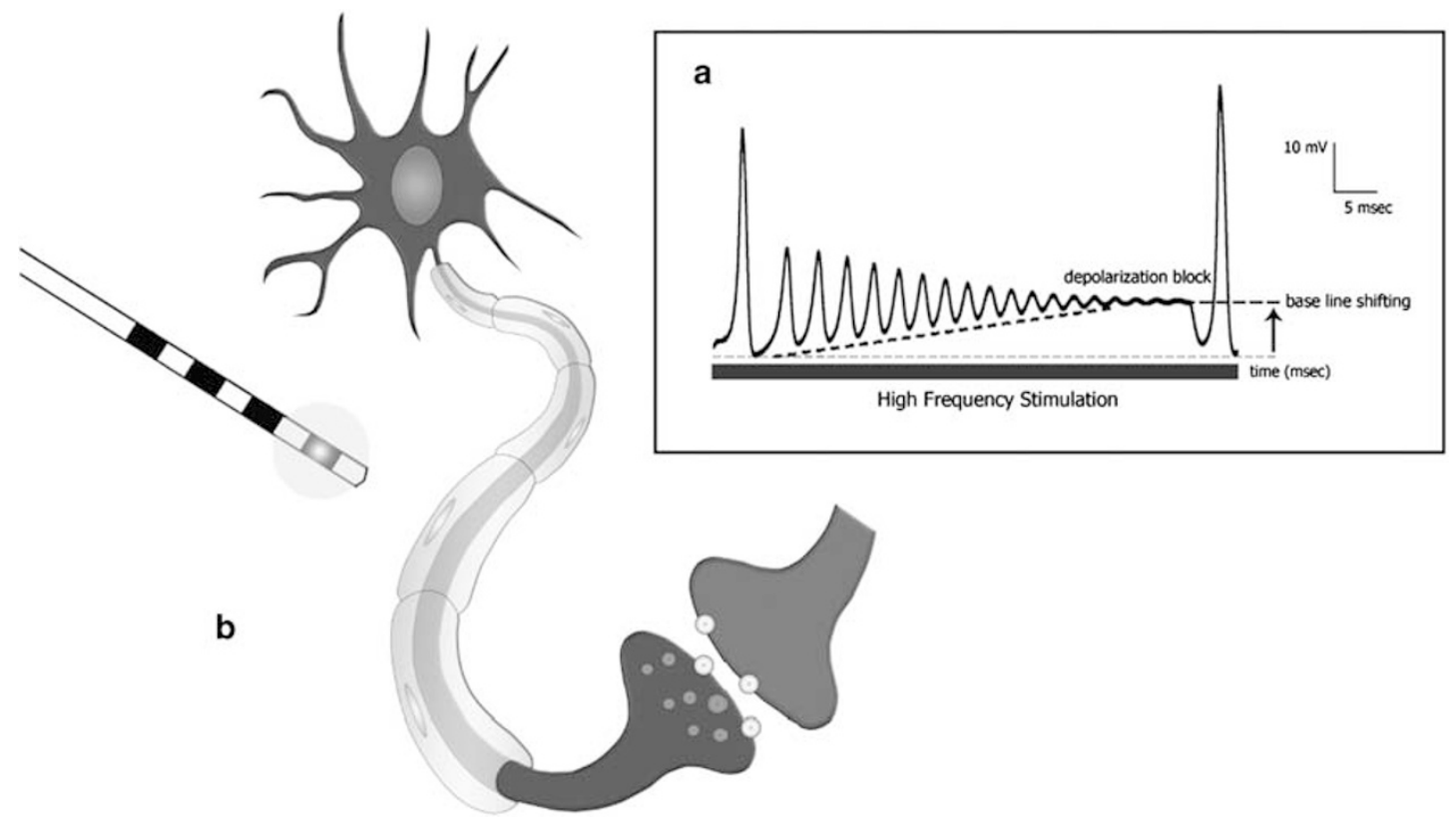

Figure 3 Principal mechanisms involved in the effects of high-frequency deep brain stimulation. (a) One of the mechanisms responsible for functional target inactivation of cell bodies is depolarization block. This state is characterized by an initial membrane depolarization, followed by a continuous reduction in the amplitude of action potentials (APs) until their complete suppression. (b) In addition to local effects, DBS has been shown to influence structures at a distance from the target. One of the principal mechanisms involved in this effect is activation of axonal pathways near the electrodes. This may induce changes in neuronal firing in structures projecting to or receiving projections from the target, neurotransmitter release, and the development of neuroplasticity. Panel (a) was modified from Florence et al (20I5) and reprinted by permission from SAGE publications.

Mechanisms responsible for the anxiolytic effects of BLA DBS are largely unknown. Under basal conditions, glutamatergic projection neurons in rodents are tonically inhibited by local GABAergic interneurons. Exposure to stress reduces this inhibitory control, enabling the firing of principal cells (Sanders and Shekhar, 1995a,b). Supporting the notion that increased BLA activity leads to anxiety, the focal administration of GABA agonists has anxiolytic effects and reduces fear responses, whereas treatments that counter BLA inhibition are largely anxiogenic (Sajdyk and Shekhar, 1997; Sanders and Shekhar, 1995a,b). This classical view, however, has been challenged by recent experiments, suggesting that the role of BLA in fear/anxiety may be far more complex. An example of this complexity is the finding that the BLA is comprised of subpopulations of 'fear' or 'extinction' neurons, which are predominantly active in situations of high fear or when fear memories are being extinguished (Senn et al, 2014; Tovote et al, 2015). In addition, excitation of parvalbumin-positive interneurons may inhibit somatostatin-expressing ones, ultimately disinhibiting BLA principal cells during the acquisition of fear memories (Wolff et al, 2014). Finally, excitation of different BLA principal cells may induce distinct behavioral effects, depending on their downstream projections. When active, efferents to the $\mathrm{CEl}$ in rodents may reduce (and not increase) anxiety via feedforward inhibition of the CEm (Tye et al, 2011).

As described above, behavioral effects of BLA DBS are largely anxiolytic, thus resembling the effects of BLA lesions or pharmacological inactivation. As such, a conceivable hypothesis to explain DBS effects is that of a stimulationinduced focal inhibition of neuronal populations. This, however, is fairly simplistic if one bears in mind the complexity of DBS mechanisms. Another scenario that could generate a net inhibitory BLA response would involve the excitation of fiber pathways innervating downstream structures that might reduce fear and anxiety (eg, to the CEm). Alternatively, DBS could enhance the activity of GABAergic presynaptic terminals to BLA principal neurons, which may ultimately reduce firing of the latter cells and modulate synaptic plasticity. With different interneurons projecting not only to different portions of BLA principal cells but also to other interneuron populations, the ultimate consequence of modulating presynaptic GABAergic projections is still unclear.

\section{Ventral Striatum}

After undergoing a 3-day fear conditioning/extinction paradigm, rats were given high-frequency stimulation into the VS for $3 \mathrm{~h}: 1 \mathrm{~h}$ before, $1 \mathrm{~h}$ during, and $1 \mathrm{~h}$ after extinction training (Rodriguez-Romaguera et al, 2012). When DBS-treated rats were considered as a single group, no differences in extinction learning were found between animals receiving active stimulation and animals receiving sham treatment. Further analysis of data, however, revealed that animals with electrodes implanted dorsal to the anterior commissure (Dorsal DBS) had significantly less freezing than sham controls, whereas those implanted with Ventral DBS electrodes had the opposite response (Rodriguez-Romaguera et al, 2012). Injections of the GABAergic agonist muscimol into these same targets did not replicate stimulation effects, suggesting that DBS-induced responses were unlikely due to a functional target inactivation (Rodriguez-Romaguera $e t$ al, 
2012). Immunocytochemical analysis revealed that the facilitation of extinction following Dorsal DBS was associated with increased levels of pERK in PL, IL, orbitofrontal cortex (OFC), and CE/ITC (Rodriguez-Romaguera et al, 2012) and Fos in PL, IL, and CE (Do-Monte et al, 2013). Regions of the VS that enhanced extinction seemed to be those receiving innervation from the OFC (Rodriguez-Romaguera et al, 2015). In the studies mentioned above, stimulation did not alter BLA immediate early gene expression. As BLA-accumbens projections are primarily involved in mechanisms of reward (Namburi et al, 2015), it is unlikely that VS DBS-induced effects on fear extinction were due to changes in the rewarding/motivational tone of the behavioral experience.

In addition to neurocircuitry changes, DBS in dorsal but not in ventral regions of the VS increased BDNF expression in PL and IL (Do-Monte et al, 2013). This suggests that stimulation-induced activation of structures at a distance from the target and associated neuroplastic changes may be the predominant mechanisms involved in the effects of VS DBS.

\section{Hippocampus}

The rodent hippocampus may be subdivided into dorsal and ventral (considered homologous to the posterior and anterior hippocampus in primates). The former receives multimodal input from entorhinal and associated cortical regions and has a crucial role in spatial learning and memory. The latter connects with the amygdala/hypothalamus and is more involved in mechanisms of anxiety, fear, and stress.

To investigate plasticity in PFC-hippocampal circuits during fear extinction, several studies using hippocampal stimulation have been conducted in rodents (Deschaux et al, 2011; Farinelli et al, 2006; Vouimba et al, 1999). Overall, high-frequency stimulation (eg, $100 \mathrm{~Hz}$ ) delivered to the hippocampus/fimbria after conditioning and/or extinction was found to enhance extinction learning and/or reduce freezing during recall sessions (Deschaux et al, 2011; Farinelli et al, 2006; Vouimba et al, 1999). Though in most cases stimulation was given for very short periods (eg, series of 100-200 pulses/few seconds; Deschaux et al, 2011; Farinelli et al, 2006; Vouimba et al, 1999), in others it was applied for $25 \mathrm{~min}$ at low frequencies (eg, $2 \mathrm{~Hz}$; Deschaux et al, 2010; Garcia et al, 2008). At these latter settings, ventral CA1 stimulation either 6 or $14 \mathrm{~h}$ following contextual conditioning reduced freezing during extinction (Cleren et al, 2013). In contrast, stimulation of the dorsal CA1 or the CA2 region in between ventral and dorsal hippocampi following extinction were shown to counter the development of plasticity and enhance freezing during extinction recall (Deschaux et al, 2010; Garcia et al, 2008). This suggests that improvement or impairment in extinction or extinction/ recall following hippocampal DBS may depend on the targeted region, stimulation parameters, and timing of stimulation delivery.

Similar to DBS, conclusions from optogenetic studies have been somewhat controversial. The inhibition (Goshen et al, 2011) or disinhibition (Lovett-Barron et al, 2014) of CA1 pyramidal cells during fear conditioning and/or recall prevented the acquisition and/or recall of contextual memories. These discrepancies suggest that for CA1 neurons 
to have a role in contextual conditioning they need to be physiologically operational. Similarly, inhibition or excitation of dentate gyrus (DG) granule cells in the dorsal hippocampus during fear conditioning impaired memory acquisition (Kheirbek et al, 2013). Altogether, optogenetic experiments support the notion that both dorsal DG and CA1 are necessary for the acquisition of contextual memory (Liu et al, 2012), whereas only CA1 pyramidal cells are involved in recall. Little evidence exists to support a role for ventral DG granule cells in the acquisition of contextual fear memories (Kheirbek et al, 2013).

In tests assessing anxiety-like behavior (eg, open field, elevated plus maze), excitation of ventral DG granule cells induced an anxiolytic effect, whereas inhibition of these same neurons had no behavioral consequences (Kheirbek et al, 2013). Dorsal DG inhibition also had no effect on baseline anxiety, whereas stimulation of this region resulted in a general increase in exploratory behavior. This suggests that, though an increase in activity in both dorsal and ventral DG may be anxiolytic, neither region seems to be crucial for the development of anxiety responses.

As for mechanisms of hippocampal DBS, the behavioral effects of stimulation applied to the hippocampus are variable and seem to depend on the region (dorsal vs ventral), subfield, frequency, and timing of current delivery. The hypothesis that stimulation-induced plasticity may be important is supported by three lines of evidence. First, optogenetic experiments reveal that hippocampal neurons need to be functionally operational for contextual fear conditioning to occur (a scenario that is not compatible with DBS-induced changes in the activity of cells bodies and fibers). Second, in most DBS studies showing a reduction in fear responses, stimulation was applied after conditioning/ extinction and not during extinction or recall. Third, the facilitatory effects of DBS on extinction and recall were largely recorded in experiments using high-frequency settings (ie, similar to those used to elicit long-term potentiation).

\section{Prefrontal Cortex}

Several studies have used electrical stimulation protocols to ascertain the role of PFC structures in fear conditioning/ extinction. Behavioral findings, however, are somewhat controversial and seem to depend on the PFC region and timing of stimulation delivery. For example, Vidal-Gonzalez et al (2006) have found that PL stimulation impaired, whereas IL stimulation facilitated extinction learning. This same group of investigators have found that the positive effects of IL stimulation were only observed when current was administered between 100 and $400 \mathrm{~ms}$ after the presentation of tones, with no effects being recorded when stimulation was delivered $1 \mathrm{~s}$ before or after CS (Milad and Quirk, 2002; Milad et al, 2004). In contrast to these findings, high-frequency stimulation (around $100 \mathrm{~Hz}$ ) delivered in pulses or for $10 \mathrm{~min}$ after conditioning/extinction and/or reconditioning was found to reduce freezing when animals were re-exposed to the conditioning context (Deschaux et al, 2011; Nachon et al, 2014; Zheng et al, 2013; Maroun et al, 2012). Thus, PFC stimulation may facilitate extinction when current is time-locked to CS or delivered at high frequencies following conditioning/extinction.
Optogenetic studies have shown that activation of IL principal cells expressing channelrhodopsin (ChR2) during extinction reduced freezing and facilitated extinction recall (Do-Monte et al, 2015). In contrast, inhibition of principal cells expressing halorhodopsin (eNpHR) during extinction did not affect fear expression but impaired the retrieval of fear memories. These results imply that IL principal cells may be involved in mechanisms that facilitate the expression of freezing but are not necessary for within-session extinction. Moreover, they corroborate previous data suggesting that IL activity during extinction training is probably necessary for later retrieval (Milad and Quirk, 2002; Milad and Quirk, 2012), an effect that may be due to the development of plasticity within the IL and downstream structures (eg, ITC and BLA; Amano et al, 2010). Though some optogenetic findings are in line with electrical stimulation experiments, a few considerations need to be made. The frequency used during optical stimulation is much lower than that commonly delivered during DBS. At $20-50 \mathrm{~Hz}$, neurons can still respond to stimulation pulses. At $130 \mathrm{~Hz}$, cell bodies are unable to do so and often undergo a state of depolarization block (Florence et al, 2015; Hamani and Temel, 2012). The reconciliation of such discrepancies may lie in the modulation of fiber pathways. As mentioned in the sections above, fibers can be driven by stimulation frequencies in the DBS range. As such, axons from IL cells undergoing depolarization block, fibers en passant, and those innervating the stimulated target would still be responsive and may be activated at $130 \mathrm{~Hz}$ (Hamani et al, 2010). Under these circumstances, the net effect of IL DBS would correspond to that of an excitation, with IL projections being tonically driven and able to release neurotransmitters, increase BDNF levels, and enhance plasticity in downstream structures (eg, brainstem, amygdala, hippocampus) as well as in the target region (Bambico et al, 2015; Hamani et al, 2010).

\section{CONCLUSIONS}

In the only case report published to date, Langevin et al (2015) have treated a combat veteran with BLA DBS. Eight months following surgery, the patient experienced a reduction of approximately $40 \%$ in PTSD symptoms. These promising results certainly require further corroboration. As for other psychiatric conditions, extensive research will be needed to establish optimal stimulation parameters, mechanisms of action, and the kinetics of a DBS response.

In animal models, electrical stimulation has been used to ascertain the role of specific structures and behavioral consequences of delivering stimulation in different protocols/preclinical models. Overall, stimulation of the BLA, VS, hippocampus, and PFC seemed to facilitate fear extinction/recall and induce anxiolytic responses in different tests. Mechanisms of these effects are largely unknown but may be related to an enhancement in plasticity and the modulation of fibers rather than to a lesion-like effect. To dissect such complex processes, optogenetics may be of use, as this technique allows an appraisal of the role played by individual cell types, projections, and other neural elements in behavioral responses. By refining the knowledge of specific elements responsible for a DBS response, we may 
not only gain further insight into how this therapy works but also help to improve it. As an example, responses dependent on neuronal firing rather than fiber activation may demand stimulation settings more suitable to depolarize cell populations (eg, lower frequencies and higher pulse width). Whether projection fibers are found to be important, new directional DBS electrodes capable of steering current to their specific anatomical location may be more appropriate.

A few caveats need to be taken into account when contemplating translation of data from animals to patients with PTSD. In experimental models, naive rodents exposed to traumatic events often undergo single behavioral testing within short intervals. Genetic variation and long-term maladaptive responses are rarely taken into account. In the clinic, surgical candidates are patients who fail multiple medical treatments. In PTSD, this subpopulation is often comprised of individuals exposed to repeated/chronic or extreme trauma, whose symptoms are not only limited to extinction deficits and anxiety but also include changes in affect regulation, attention, memory, and self-perception, as well as difficulties with interpersonal relationships. Though DBS may help to treat fear extinction and anxiety, it is unclear whether other aspects of PTSD will improve as well.

Despite these caveats, studies in animal models may certainly bring an important contribution to the field (Hamani and Temel, 2012). With a good predictive and construct validity in many neuropsychiatric applications of DBS (Hamani and Temel, 2012), preclinical models may help us further understand mechanisms of this therapy, explore new applications and targets, and understand the kinetics involved in stimulation-induced behavioral responses. As in other fields of psychiatry, additional studies are certainly required for a better appraisal of how DBS exerts its effects, so that we may perfect the clinical applications of this technique in PTSD-like states.

\section{FUNDING AND DISCLOSURE}

The authors declare no conflict of interest.

\section{ACKNOWLEDGMENTS}

We thank Danilo Barbosa for the illustrations in Figures 1 and 3. A list of references not included in the main text may be found in the Supplementary Materials.

\section{REFERENCES}

Amano T, Unal CT, Pare D (2010). Synaptic correlates of fear extinction in the amygdala. Nat Neurosci 13: 489-494.

Andero R, Ressler KJ (2012). Fear extinction and BDNF: translating animal models of PTSD to the clinic. Genes Brain Behav 11: 503-512.

Bambico FR, Bregman T, Diwan M, Li J, Darvish-Ghane S, Li Z et al (2015). Neuroplasticity-dependent and -independent mechanisms of chronic deep brain stimulation in stressed rats. Transl Psychiatry 5: e674.

Burgos-Robles A, Vidal-Gonzalez I, Quirk GJ (2009). Sustained conditioned responses in prelimbic prefrontal neurons are correlated with fear expression and extinction failure. J Neurosci 29: 8474-8482.
Cleren C, Tallarida I, Guiniec EL, Janin F, Nachon O, Canini F et al (2013). Low-frequency stimulation of the ventral hippocampus facilitates extinction of contextual fear. Neurobiol Learn Mem 101: 39-45.

Deschaux O, Motanis H, Spennato G, Moreau JL, Garcia R (2011). Re-emergence of extinguished auditory-cued conditioned fear following a sub-conditioning procedure: effects of hippocampal and prefrontal tetanic stimulations. Neurobiol Learn Mem 95: 510-518.

Deschaux O, Thevenet A, Spennato G, Arnaud C, Moreau JL, Garcia R (2010). Low-frequency stimulation of the hippocampus following fear extinction impairs both restoration of rapid eye movement sleep and retrieval of extinction memory. Neuroscience 170: 92-98.

Do-Monte FH, Manzano-Nieves G, Quinones-Laracuente K, Ramos-Medina L, Quirk GJ (2015). Revisiting the role of infralimbic cortex in fear extinction with optogenetics. J Neurosci 35: 3607-3615.

Do-Monte FH, Rodriguez-Romaguera J, Rosas-Vidal LE, Quirk GJ (2013). Deep brain stimulation of the ventral striatum increases BDNF in the fear extinction circuit. Front Behav Neurosci 7: 102.

Eysenck HJ (1979). Behavioral therapy and the philosophers. Behav Res Ther 17: 511-514.

Farinelli M, Deschaux O, Hugues S, Thevenet A, Garcia R (2006). Hippocampal train stimulation modulates recall of fear extinction independently of prefrontal cortex synaptic plasticity and lesions. Learn Mem 13: 329-334.

Florence G, Sameshima K, Fonoff ET, Hamani C (2015). Deep brain stimulation: more complex than the inhibition of cells and excitation of fibers. Neuroscientist (e-pub ahead of print).

Foa EB (2000). Psychosocial treatment of posttraumatic stress disorder. J Clin Psychiatry 61 (Suppl 5): 43-48 (discussion 49-51).

Garcia R, Spennato G, Nilsson-Todd L, Moreau JL, Deschaux O (2008). Hippocampal low-frequency stimulation and chronic mild stress similarly disrupt fear extinction memory in rats. Neurobiol Learn Mem 89: 560-566.

Goshen I, Brodsky M, Prakash R, Wallace J, Gradinaru V, Ramakrishnan C et al (2011). Dynamics of retrieval strategies for remote memories. Cell 147: 678-689.

Hamani C, Diwan M, Macedo CE, Brandao ML, Shumake J, Gonzalez-Lima F et al (2010). Antidepressant-like effects of medial prefrontal cortex deep brain stimulation in rats. Biol Psychiatry 67: 117-124.

Hamani C, Temel Y (2012). Deep brain stimulation for psychiatric disease: contributions and validity of animal models. Sci Transl Med 4: 142rv148.

Hashimoto T, Elder CM, Okun MS, Patrick SK, Vitek JL (2003). Stimulation of the subthalamic nucleus changes the firing pattern of pallidal neurons. J Neurosci 23: 1916-1923.

Kheirbek MA, Drew LJ, Burghardt NS, Costantini DO, Tannenholz L, Ahmari SE et al (2013). Differential control of learning and anxiety along the dorsoventral axis of the dentate gyrus. Neuron 77: 955-968.

Langevin JP, De Salles AA, Kosoyan HP, Krahl SE (2010). Deep brain stimulation of the amygdala alleviates post-traumatic stress disorder symptoms in a rat model. J Psychiatr Res 44: 1241-1245.

Langevin JP, Koek RJ, Schwartz HN, Chen JW, Sultzer DL, Mandelkern MA et al (2015). Deep brain stimulation of the basolateral amygdala for treatment-refractory posttraumatic stress disorder. Biol Psychiatry (e-pub ahead of print).

Laurent V, Westbrook RF (2009). Inactivation of the infralimbic but not the prelimbic cortex impairs consolidation and retrieval of fear extinction. Learn Mem 16: 520-529.

Liu X, Ramirez S, Pang PT, Puryear CB, Govindarajan A, Deisseroth $\mathrm{K}$ et al (2012). Optogenetic stimulation of a hippocampal engram activates fear memory recall. Nature 484: 381-385. 
Lovett-Barron M, Kaifosh P, Kheirbek MA, Danielson N, Zaremba JD, Reardon TR et al (2014). Dendritic inhibition in the hippocampus supports fear learning. Science 343: 857-863.

Lozano AM, Dostrovsky J, Chen R, Ashby P (2002). Deep brain stimulation for Parkinson's disease: disrupting the disruption. Lancet Neurol 1: 225-231.

Maren S, Quirk GJ (2004). Neuronal signalling of fear memory. Nat Rev Neurosci 5: 844-852.

Maroun M, Kavushansky A, Holmes A, Wellman C, Motanis H (2012). Enhanced extinction of aversive memories by high-frequency stimulation of the rat infralimbic cortex. PLoS One 7: e35853.

Milad MR, Quirk GJ (2002). Neurons in medial prefrontal cortex signal memory for fear extinction. Nature 420: 70-74.

Milad MR, Quirk GJ (2012). Fear extinction as a model for translational neuroscience: ten years of progress. Annu Rev Psychol 63: 129-151.

Milad MR, Vidal-Gonzalez I, Quirk GJ (2004). Electrical stimulation of medial prefrontal cortex reduces conditioned fear in a temporally specific manner. Behav Neurosci 118: 389-394.

Moser A, Gieselberg A, Ro B, Keller C, Qadri F (2003). Deep brain stimulation: response to neuronal high frequency stimulation is mediated through $\mathrm{GABA}(\mathrm{A})$ receptor activation in rats. Neurosci Lett 341: 57-60.

Nachon O, Cleren C, Husson S, Huguet C, Auclair J, Faure S et al (2014). Prefrontal tetanic stimulation, following fear reconditioning, facilitates expression of previously acquired extinction. Neurobiol Learn Mem 113: 62-68.

Namburi P, Beyeler A, Yorozu S, Calhoon GG, Halbert SA, Wichmann $\mathrm{R}$ et al (2015). A circuit mechanism for differentiating positive and negative associations. Nature 520: 675-678.

Ranck JB Jr. (1975). Which elements are excited in electrical stimulation of mammalian central nervous system: a review. Brain Res 98: 417-440.

Rodriguez-Romaguera J, Do Monte FH, Quirk GJ (2012). Deep brain stimulation of the ventral striatum enhances extinction of conditioned fear. Proc Natl Acad Sci USA 109: 8764-8769.

Rodriguez-Romaguera J, Do-Monte FH, Tanimura Y, Quirk GJ, Haber SN (2015). Enhancement of fear extinction with deep brain stimulation: evidence for medial orbitofrontal involvement. Neuropsychopharmacology 40: 1726-1733.

Sajdyk TJ, Shekhar A (1997). Excitatory amino acid receptors in the basolateral amygdala regulate anxiety responses in the social interaction test. Brain Res 764: 262-264.

Saldivar-Gonzalez JA, Posadas-Andrews A, Rodriguez R, Gomez C, Hernandez-Manjarrez ME, Ortiz-Leon S et al (2003). Effect of electrical stimulation of the baso-lateral amygdala nucleus on defensive burying shock probe test and elevated plus maze in rats. Life Sci 72: 819-829.
Sanders SK, Shekhar A (1995a). Anxiolytic effects of chlordiazepoxide blocked by injection of GABAA and benzodiazepine receptor antagonists in the region of the anterior basolateral amygdala of rats. Biol Psychiatry 37: 473-476.

Sanders SK, Shekhar A (1995b). Regulation of anxiety by GABAA receptors in the rat amygdala. Pharmacol Biochem Behav 52: 701-706.

Senn V, Wolff SB, Herry C, Grenier F, Ehrlich I, Grundemann J et al (2014). Long-range connectivity defines behavioral specificity of amygdala neurons. Neuron 81: 428-437.

Sierra-Mercado D, Padilla-Coreano N, Quirk GJ (2011). Dissociable roles of prelimbic and infralimbic cortices, ventral hippocampus, and basolateral amygdala in the expression and extinction of conditioned fear. Neuropsychopharmacology 36: $529-538$.

Stidd DA, Vogelsang K, Krahl SE, Langevin JP, Fellous JM (2013). Amygdala deep brain stimulation is superior to paroxetine treatment in a rat model of posttraumatic stress disorder. Brain Stimul 6: 837-844.

Sui L, Huang S, Peng B, Ren J, Tian F, Wang Y (2014). Deep brain stimulation of the amygdala alleviates fear conditioning-induced alterations in synaptic plasticity in the cortical-amygdala pathway and fear memory. J Neural Transm 121: 773-782.

Tovote P, Fadok JP, Luthi A (2015). Neuronal circuits for fear and anxiety. Nat Rev Neurosci 16: 317-331.

Tye KM, Prakash R, Kim SY, Fenno LE, Grosenick L, Zarabi H et al (2011). Amygdala circuitry mediating reversible and bidirectional control of anxiety. Nature 471: 358-362.

VanElzakker MB, Dahlgren MK, Davis FC, Dubois S, Shin LM (2014). From Pavlov to PTSD: the extinction of conditioned fear in rodents, humans, and anxiety disorders. Neurobiol Learn Mem 113: 3-18.

Vidal-Gonzalez I, Vidal-Gonzalez B, Rauch SL, Quirk GJ (2006). Microstimulation reveals opposing influences of prelimbic and infralimbic cortex on the expression of conditioned fear. Learn Mem 13: 728-733.

Vouimba RM, Garcia R, Jaffard R (1999). Pretraining tetanic fimbrial stimulation impairs the expression but not the acquisition of contextual fear conditioning in mice. Neuroscience 93: 869-876.

Wolff SB, Grundemann J, Tovote P, Krabbe S, Jacobson GA, Muller C et al (2014). Amygdala interneuron subtypes control fear learning through disinhibition. Nature 509: 453-458.

Zheng X, Deschaux O, Lavigne J, Nachon O, Cleren C, Moreau JL et al (2013). Prefrontal high-frequency stimulation prevents subconditioning procedure-provoked, but not acute stress-provoked, reemergence of extinguished fear. Neurobiol Learn Mem 101: $33-38$.

Supplementary Information accompanies the paper on the Neuropsychopharmacology website (http://www.nature.com/npp) 